\title{
Constraints on the gamma-ray opacity of the Universe with the Fermi-LAT instrument
}

\author{
Anita Reimer*i \\ Institut für Theoretische Physik, Universität Innsbruck, Austria \\ E-mail: anita.reimer@uibk.ac.at
}

\author{
A. Bouvier ${ }^{1}$, A. Chen ${ }^{2}$, S. Raino ${ }^{3}$, S. Razzaque ${ }^{4}$, L. Reyes ${ }^{5}$, for the Fermi-LAT \& GBM \\ collaboration \\ ${ }^{1}$ Santa Cruz Institute for Particle Physics, University of California, Santa Cruz, USA \\ ${ }^{2}$ INAF - Instituto di Astrofisica Spaziale d Fisica Cosmica, Milano, Italy \\ ${ }^{3}$ Dipartimento di Fisica "M. Merlin" dell'Universita d del Politecnico di Bari, Italy \\ ${ }^{4}$ Space Science Division, Naval Research Laboratory, Washington DC, USA \\ ${ }^{5}$ Kavli Institute for Cosmology, University of Chicago, USA
}

\begin{abstract}
The Fermi Large Area Telescope is scanning the sky for gamma-rays in the range $20 \mathrm{MeV}$ to over $300 \mathrm{GeV}$, revealing a wealth of high energy sources. Gamma rays may interact with low-energy photons from the Extragalactic Background Light (EBL) through photon-photon pair production if above the energy threshold. This results in redshift- and energy-dependent attenuation features in extragalactic source spectra such as from blazars and Gamma-Ray Bursts (GRBs). Using photons above $10 \mathrm{GeV}$ collected by the LAT during more than one year of observations from high-redshift blazars and GRBs, the effect of gamma-ray flux attenuation by the EBL is investigated. I present several techniques that are used to place robust upper limits on the gamma-ray opacity of the Universe at various energies and redshifts. The comparison with predictions from a range of EBL models allows then to derive high-confidence limits on EBL intensity models. In particular, the results of this analysis imply that the EBL intensity at optical/UV wavelengths must be significantly lower as that predicted by the "baseline model".
\end{abstract}

Cosmic Radiation Fields: Sources in the early Universe

November 9-12, 2010

Desy, Germany

\footnotetext{
* Speaker.

${ }^{\dagger}$ AR acknowledges support by the Marie Curie IRG grant 248037 within the FP7 Program.
} 


\section{Introduction}

Gamma rays are known for their power to penetrate deeply into materials. However as they propagate through the Universe they are subject to absorption when traversing dense radiation fields via photon-photon pair production if above the pair production threshold. Because of the strongly peaked cross section close to threshold, more than half the interactions occur in a small energy range $\Delta \varepsilon \approx(4 / 3 \pm 2 / 3) \varepsilon$ centered around the comoving energy $\varepsilon \approx 0.8(E / T e V)^{-1} \mathrm{eV}$ for a smooth target photon field of density $n(\varepsilon)$. Photons above $\sim 10 \mathrm{GeV}$ up to $\mathrm{TeV}$ energies are therefore absorbed primarily in the IR-to-optical/UV background radiation field, the Extragalactic Background Light (EBL). The $\gamma$ - ray spectrum as measured on Earth appears distorted when compared to the emitted spectrum: $F_{o b s}(E)=F_{\text {emit }}(E(1+z)) \times \exp \left(-\tau_{\gamma \gamma}(E, z)\right)$ where $F_{o b s}$ and $F_{\text {emit }}$ is the energy dependent observed and emitted flux, and $\tau_{\gamma \gamma}(E, z)$ is the energy $E$ and redshift $z$ dependent optical depth to $\gamma \gamma$ pair production. The value of the optical depth depends on the $\gamma \gamma$ pair production cross section, the distance the $\gamma$ - rays have traversed, and the density of the target photon field. Thus, by comparing the observed and emitted spectrum (if known) of a source at known redshift $z$, information about the intervening target radiation field, the EBL, can be aquired. This is interesting because direct measurements of its intensity are difficult, and the EBL has imprinted valuable information about star formation rates, stellar radiation and dust extinction processes accumulated over cosmological time.

The interrelationship between optical depth $\tau_{\gamma \gamma}$, energy and redshift can be represented in a cube. Slices along different planes of this cube then allow to deduce information about relevant observables for this kind of analysis of $\gamma$-ray measurements. For example, by cutting at $\tau_{\gamma \gamma}(E, z)=1$ one gets the e-folding cutoff energy versus redshift. A quick comparison with opacity expectations from EBL models then provides hints about the relevant redshift range where significant distortions in $\gamma$ - ray spectra can be expected for a given energy range. Distortion signatures in spectra measured in the LAT energy range are expected for sources at redshift $z \geq 0.5$. A cut at fixed redshift $z$ gives the expected optical depth as a function of (observed) energy $E$. Estimating the opacity

$$
\tau_{\gamma \gamma}(E, z)=\ln \left(F_{\text {emit }}(E(1+z)) / F_{\text {obs }}(E)\right)
$$

from a source spectrum at that redshift $z$ requires the knowledge of both, the observed and emitted spectrum. This kind of analysis has been performed in the past by the modern Air Cherenkov Telescopes (ACTs) in the $\mathrm{TeV}$ band by fixing the intrinsically emitted spectrum (assumed following a power law $F_{\text {emit }} \propto E^{-\Gamma_{\text {int }}}$ ) to reasonable values for $\Gamma_{\text {int }}$ (e.g., Aharonian et al. 2006). Since the Fermi-LAT energy range covers both, the absorbed part of the spectrum $\geq 10 \mathrm{GeV}$ and the unabsorbed part $(<10 \mathrm{GeV})$, more stringent limits on the opacity of $\gamma$-rays can in principle now be deduced. Adding the GeV energy range therefore not only allows to probe (for the first time by $\gamma$ ray measurements) the UV band of the EBL, and its evolution, but also extends the lever arm into the important unabsorbed energy range thereby uncovering the intrinsic source spectrum.

Here I report on limits on the opacity of tens to hundred of $\mathrm{GeV}$ photons while propagating cosmological distances through the Universe which have recently been derived by the Fermi-LAT instrument. The reader may consult Atwood et al. (2009) for details on the Fermi-LAT instrument. 


\section{Active Galactic Nuclei (AGN) and Gamma Ray Bursts (GRBs) as opacity probes}

Probing the $\mathrm{GeV}$ opacity of the Universe requires bright (such to allow a detailed spectral analysis) yet distant $(z \geq 0.5) \gamma$-ray sources with a reliably known redshift and whose photon spectrum extends significantly beyond $\sim 10 \mathrm{GeV}$. These properties are found among the objects in the blazar class, the most numerous extragalactic $\gamma$-ray emitting source population observed so far, and some LAT-detected GRBs. To evaluate the influence of any possible biases on the analysis a good understanding of the intrinsic properties of these source populations is needed.

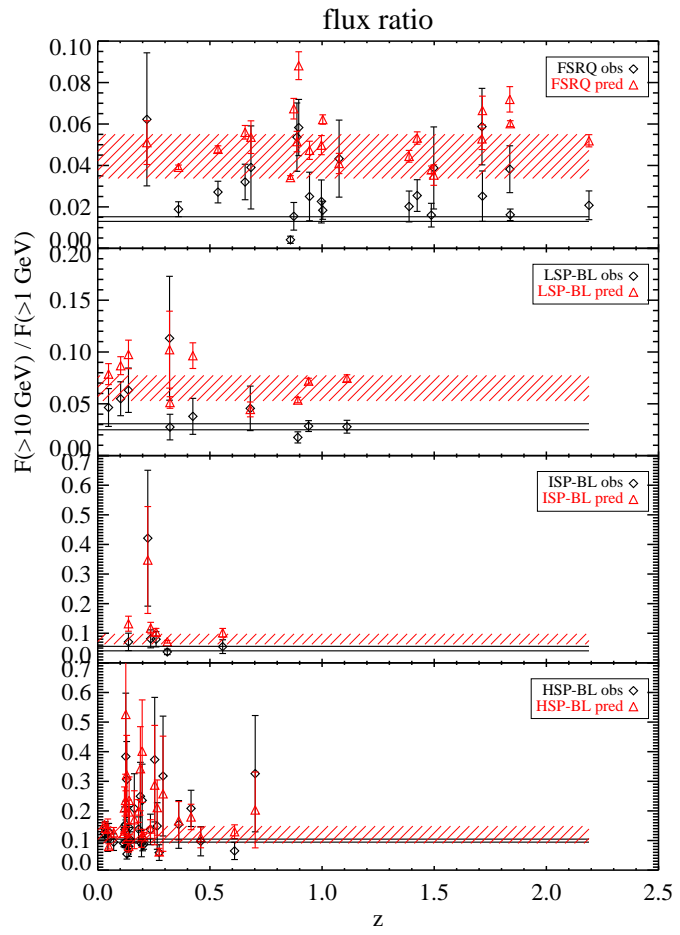

Figure 1: The evolution of $F_{>10 \mathrm{GeV}} / F_{>1 \mathrm{GeV}}$ shows no statistically significant trend within each blazar class (see text for details).
Delayed $\mathrm{GeV}$ emission has been detected by now from a number of LAT GRBs in their prompt phase. Its spectrum mostly follows a hard power law (photon spectral index $\Gamma \sim 1.5-2)$ at $>100 \mathrm{MeV}$, and adds to the usual band function in the keV-MeV band. Since some bursts show significant spectral evolution during the first seconds after the trigger, the following GRB spectral analysis is restricted to a sufficient small time window. LAT GRBs detected up to 30 September 2009 are considered. Details of the analysis can be found in Abdo et al. (2010c). As summarized in the 1LAC catalog (Abdo et al 2010a) the first 11 months of the mission brought more than 1000 significantly (TS $>25)$ detected, high-latitude $\left(|b|>10^{\circ}\right)$ $\mathrm{GeV}$ sources of which $\sim 700$ have been associated with AGNs: $\sim 42 \%$ turned out to be FSRQs, $\sim 42 \%$ BL Lacs and $16 \%$ are of unknown or other type.

The BL Lac class itself is divided up into low- (LSP), intermediate (ISP) and high- (HSP) synchrotron peaked blazars using their low energy broadband spectral energy distribution (SED). Detailed population studies of those have shown that both the $\gamma$-ray spectral index and redshift range is a distinctive feature of the source type: FSRQs possess typically soft spectra (mean $\Gamma_{F S R Q} \sim 2.3$ ) and exist preferentially at high redshifts. As one goes along the blazar sequence FSRQ-LSPISP-HSP the mean spectral shape becomes more and more hard (mean $\Gamma_{L S P} \sim 2.2, \Gamma_{I S P} \sim 2.1$, $\Gamma_{H S P} \sim 1.9$ ), and the redshift range narrows and shifts to smaller distances (Abdo et al. 2009, 2010b). The resulting spectral index-redshift relation is an intrinsic property of the blazar population, in particular not caused primarily by EBL absorption. Furthermore, most bright FSRQs and some LSPs show source-intrinsic breaks at $\sim 1-10 \mathrm{GeV}$ (galaxy frame) whereas most HSPs do not show any deviation from straight power-laws at LAT energies. These population properties turn out crucial for understanding the results of the flux-ratio method (Chen et al. 2004), a population based technique designed to uncover EBL-caused absorption in a large sample of blazars. 


\section{Flux ratios - a population based method}

Here the redshift-dependence of the $F_{>10 \mathrm{GeV}} / F_{>1 \mathrm{GeV}}$ flux ratio is proposed as a signature of EBL-caused absorption. Since this method assumes that the whole population follows a single luminosity function and spectral index distribution, it has been applied to the various blazar classes separately to avoid biases. Only clean AGN associations from the 1LAC have been used, and where flux determinations $>10 \mathrm{GeV}$ and $>1 \mathrm{GeV}$ exist. In addition to the observed ratio, also the predicted flux ratio assuming an unbroken power law and no EBL attenuation is calculated. One finds (see Fig. 1) along the sequence FSRQ-LSP-ISP-HSP that a) the redshift range becomes narrower, b) the spectra on average become harder, c) the observed and predicted flux ratios increase, and d) the difference between the predicted and observed flux ratio decreases. Points (a) and (b) are properties intrinsic to the blazar population as discussed above, the same is true for point (c) considering that the flux ratio is a measure of the spectral index, and point (d) arises from the LAT energy range moving through different parts of the blazar SED. Hence, all those findings can be explained by population intrinsic effects, hence lack any EBL induced attenuation signatures. Furthermore, no spectral evolution within each blazar class is found. The search for any EBL induced signatures by the flux ratio method remains inconclusive (see Fig. 1). See Abdo et al.(2010c) for details.

\section{Searching for EBL induced signatures in individual source spectra}

The methods discussed in the following are based on the highest energy photons (HEP) detected in LAT source spectra, and on an application of the likelihood ratio technique (LRT).

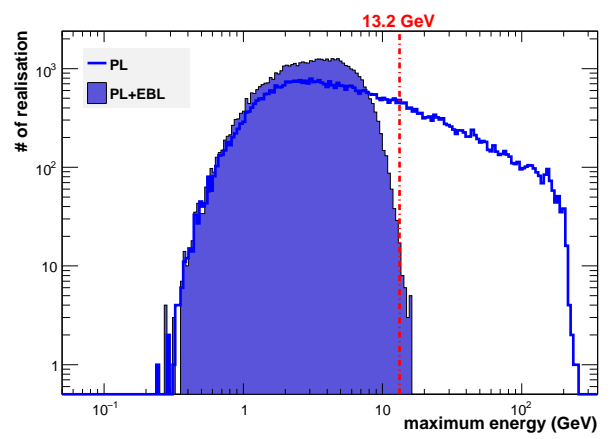

Figure 2: Distribution of the HEP from simulations $\left(10^{5}\right.$ realizations) for GRB080916C using an estimated intrinsic power law spectrum with photon index $2.15 \pm 0.22$ folded with (hatched area) and without EBL attenuation using the baseline model (see text for details).

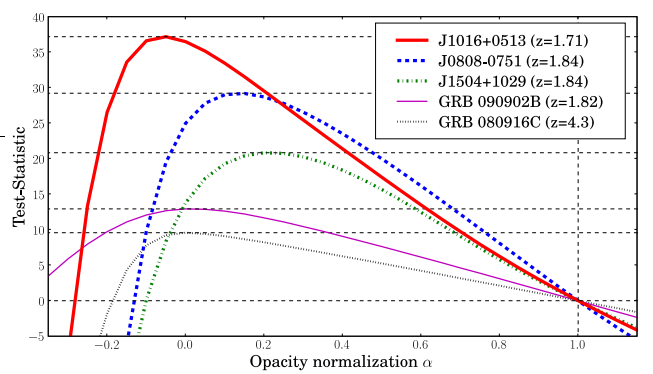

Figure 3: TS as a function of optical depth normalization $\alpha$ as a result of the LRT analysis of HEP-set sources (see text and Abdo et al. (2010c) for details).

Consider the highest energy photon (HEP) associated with an individual source (from the 1LAC and the sample of LAT detected GRBs) at redshift $z$ within the $68 \%$ containment radius of each source position. Five AGN and two GRBs turn out to have a HEP energy $E_{\text {max }}$-redshift combination which significantly lies above the $\tau_{\gamma \gamma}=3$ line of the "baseline EBL model" of Stecker et al. (2006). This model is among those which predict the highest EBL photon density, and 
will be confronted with LAT observations in the following. EBL models of lower intensity in the UV range can to date not be constrained by the 11 months LAT data. The seven sources, with $z \sim 1-4.5$ and $E_{\max } \sim 13-74 \mathrm{GeV}$, constitute the "HEP-set". All HEP-set AGN turn out to be FSRQs. The probability of non-association, $P_{b k g}$, is computed from the expected number of diffuse and instrumental background photons. The HEP method then evaluates the overall chance probability $P_{H E P}$ of detecting a source photon within the PSF $68 \%$ containment radius with $E \geq$ $E_{\text {max }}$ given a source intrinsic spectrum and the specific EBL model. Because EBL attenuation is negligible below $\sim 10 \mathrm{GeV}$ for $z \geq 3$ a conservative limit ${ }^{1}$ on the intrinsic source spectrum at high energies $>10 \mathrm{GeV}$ can be derived by extrapolating the low energy unabsorbed part into the energy range where EBL absorption is expected. One implicitly assumes here that the source intrinsic spectrum does not show any spectral upturn beyond in the energy range $>10 \mathrm{GeV}$. Notably, this is the only assumption made for the following analysis that probe the "baseline model" of Stecker et al. (2006). All but one spectrum of the HEP-set sources turn out to be best represented by simple power-laws (for J1504+1029 a log-parabolic shape is preferred). The calculation of the chance probability $P_{H E P}$ is done on the basis of Monte Carlo simulations. Fig. 2 shows as an example of the resulting distribution of HEP events for GRB080916C with the observed HEP event indicated. Notably, for the AGN in the HEP-set $P_{H E P}$ lies at the same order of magnitude as $P_{b g k}$ whereas for the GRBs $P_{b k g} \ll P_{H E P}$. Finally, the overall rejection probability $P_{r e j}$ includes the possibility that the HEP may be a background event, or may be not absorbed. For those sources with more than one photon $>10 \mathrm{GeV}$ (in three cases) the probabilities $P_{r e j}$ for each photon are combined using Fisher's method (Fisher 1925). The resulting probabilities of rejecting the "baseline model" lie up to $4.5 \sigma$. See Abdo et al (2010c) for details on this method.

The likelihood ratio technique compares the likelihood $L_{0}$ of the Null-hypothesis with a model of likelihood $L_{1}$ which best represents the data. For $F_{\text {obs }}(E)=F_{\text {emit }}(E(1+z)) \times \exp \left(-\alpha \tau_{\gamma \gamma, \text { model }}(E, z)\right)$ with $\tau_{\gamma \gamma \text {,model }}(E, z)$ the opacity predicted by the baseline model and $F_{\text {emit }}$ the power-law intrinsic spectrum, $\alpha=1$ represents the Null-hypothesis. The likelihood computations include all photons within a several degrees large region from the source on an event-by-event basis with each event weighted corresponding to the angular distance to the source, the probability of being a background photon is also included on an event-by-event basis. Defining the test statistic $T S=-2 \times\left(\log \left(L_{0}\right)-\log \left(L_{1}\right)\right)$, the best-fit $\alpha$-value is reached at the TS-maximum $T S_{\max }$. Fig. 3 shows examples of the TS-values as a function of $\alpha$. It is then straight forward to convert $\Delta T S=T S_{\max }-T S(\alpha=1)$ into the associated confidence to reject the EBL model under consideration. From Fig. 3 the highest rejection significance $(\sim 6 \sigma)$ is reached by J1016+0513 at redshift $z=1.71$. The LRT technique turned out slightly more constraining than the HEP method. The a priori choice of the considered region around the source used by the HEP method (68\% PSF containment radius) may be partly the reason. Details are in Abdo et al. (2010c).

After including multi-trial effects and combining the rejection significances from the individual sources, one finds an overall high confidence (HEP: $\sim 8.9 \sigma$, LRT: $\sim 11.4 \sigma$ ) of rejecting EBL models that predict opacities in the $20-50 \mathrm{GeV}$ energy and $z \sim 1-4$ redshift range as great as the "baseline model" of Stecker et al. (2006). This applies also to the "fast-evolution" model of

\footnotetext{
${ }^{1}$ Source intrinsic curvature cannot be decoupled from EBL-caused curvature. The so derived limits therefore include the sum of all effects that may cause curvature, and is thus a conservative limit on EBL-induced attenuation.
} 


\section{J1504+1029 -- Redshift: 1.84}

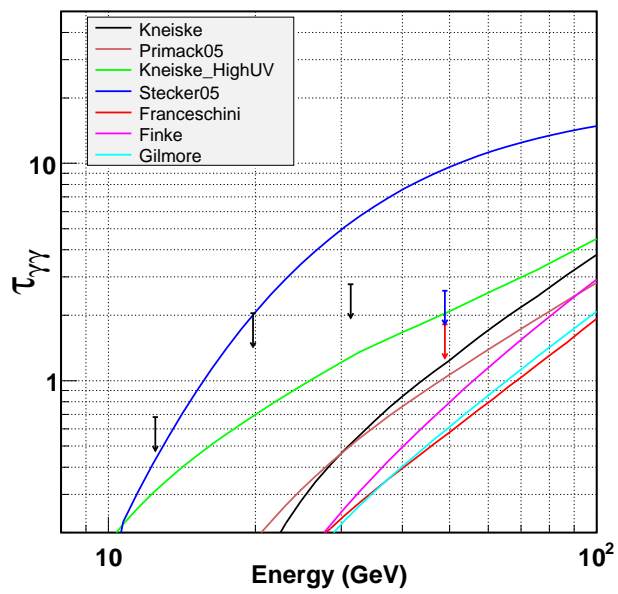

Figure 4: Optical depth upper limits for FSRQ PKS 1502+106. Black arrows: 95\% CL in all energy bins that have been used to determine the observed flux above $10 \mathrm{GeV}$, red/blue arrow: 95\%/99\% CL for the highest energy photon (see Abdo et al. 2010c for details).
Stecker et al. (2006) since it predicts even greater optical depths. Upper limits on the optical depth for all AGN in the HEP-set are derived by comparing the observed flux at various energies $>10 \mathrm{GeV}$ with that emitted by the source at redshift $z$ following Eq. 1. An upper limit of the emitted flux is estimated by extrapolating the low energy $<10 \mathrm{GeV}$ part of the spetrum to high energies, again implicitly assuming no spectral upturn of the source-intrinsic spectrum. The parameter uncertainties in the fitted flux are propagated into the optical depth upper limit computation. As an example Fig. 4 shows the resulting optical depth estimates for J1504+1029 (=PKS 1502+106). For details see Abdo et al.(2010c). The results of this analysis applied to all AGN of the HEPset is in overall agreement with those derived from the HEP and LRT method.

\section{Discussion and Conclusion}

The opacity constraints derived from the analysis of high energy photons above $10 \mathrm{GeV}$ from AGN and GRBs collected by the Fermi-LAT over more than one year of observations disfavor EBL models that predict an EBL intensity as great as that suggested by the "baseline" and "fastevolution" models of Stecker et al. (2006). Those constraints concern the optical-UV light production over cosmological time (up to $z \sim 4$ ), and hence are in principle tied to the global star formation rate density that is thought to peak around $z \sim 1$. The models of Stecker et al. (2006) belong to the class of backward evolution models of galaxy formation. Specifically here, the $60 \mu \mathrm{m}$ luminosity function plus a power law luminosity evolution has been used together with an analytic approximation for galaxy SEDs linking the $60 \mu \mathrm{m}$ range to UV wavelengths to predict the optical/UV EBL intensity. ${ }^{2}$ In particular, no star formation rate density has been used here. On the other side, the LAT-data do not yet constrain the "high-UV model"3 of Kneiske et al. (2004) with high significance where the UV background intensity has been increased by a factor of 4 with respect to their "best-fit model" that is based on a reasonable star formation rate density. Therefore the presented constraints on the $\gamma$-ray opacity from the Fermi-LAT observations can not yet place limits on the star formation rate history that are competitive with current observational estimates.

For the first time GRBs have been used here sucessfully as probes of the $\gamma$-ray opacity of the Universe. Since the GRB population is known to extend to extremely high redshifts, they constitue

\footnotetext{
${ }^{2}$ Those models don't include photon absorption by dust, possibly among the reasons for the high predicted UV EBL intensity.

${ }^{3}$ designed to take into account results derived from the proximity effect (see Kneiske et al. (2004) for details)
} 
an interesting $\gamma$-ray source class for future analysis to understand the evolution of the EBL intensity.

\section{References}

[1] Abdo, A. A., et al. 2009, ApJ 700597.

[2] Abdo, A. A., et al. 2010a, ApJ 715429.

[3] Abdo, A. A., et al. 2010b, ApJ 7101271.

[4] Abdo, A. A., et al. 2010c, ApJ 7231082.

[5] Aharonian, F. A., et al. 2006, Nature 4401018.

[6] Atwood, W. B., et al. 2009, ApJS 6971071.

[7] Chen, A., Reyes, L. C., \& Ritz, S. 2004, ApJ 608686.

[8] Fisher, R.A. 1925, Statistical Methods for Research Workers, Oliver and Boyd (Edinburgh).

[9] Kneiske, T. M., Bretz, T., Mannheim, K. \& Hartmann, D. H. 2004, A\&A 413807.

[10] Stecker, F. W., Malkan, M. A. \& Scully, S. T. 2006, ApJ 648 774, and Erratum: ApJ 6581392 (2007). 